\title{
An ab Initio Study of Sulfinic Acid and Related Species
}

\author{
Dianna Laakso and Paul Marshall* \\ Department of Chemistry, University of North Texas, PO Box 5068, Denton, Texas 76203-5068 \\ (Received: September 16, 1991)
}

\begin{abstract}
Geometries and vibrational frequencies of five isomers of $\mathrm{H}_{2} \mathrm{SO}_{2}$ have been derived at the $\mathrm{HF} / 3-21 \mathrm{G}\left({ }^{*}\right)$ and $\mathrm{MP} 2 / 3-21 \mathrm{G}\left({ }^{*}\right)$ levels of theory, and energies were obtained using MP4/6-31 $\mathrm{G}^{*} / / \mathrm{MP} 2 / 3-21 \mathrm{G}\left({ }^{*}\right)$ theory. In order of decreasing stability the isomers are $\mathrm{S}(\mathrm{OH})_{2}, \mathrm{HSO}_{2} \mathrm{H}$ (sulfinic acid, two conformers), sulfone, $\mathrm{HSOOH}$, and $\mathrm{H}_{2} \mathrm{SOO}$. Pathways leading to $\mathrm{HSO}_{2} \mathrm{H}$ formation were analyzed to determine why the more stable $\mathrm{S}(\mathrm{OH})_{2}$ was not seen in matrix-isolation experiments. The barrier to isomerization between the two isomers was estimated to be $190 \mathrm{~kJ} \mathrm{~mol}^{-1}$ relative to $\mathrm{HSO}_{2} \mathrm{H}$, and the stability of $\mathrm{HSO}_{2} \mathrm{H}$ with respect to dissociation was also considered.
\end{abstract}

\section{Introduction}

Molecules with the stoichiometry $\mathrm{H}_{2} \mathrm{SO}_{2}$ have been proposed as intermediates in the oxidation of $\mathrm{H}_{2} \mathrm{~S},{ }^{1}$ but there is little experimental information available about these species despite their possible importance in the atmospheric chemistry of sulfur. Accordingly, we have extended an earlier theoretical study ${ }^{2}$ of $\mathrm{HSO}_{2}$ and $\mathrm{HOSO}$ to several $\mathrm{H}_{2} \mathrm{SO}_{2}$ isomers, in order to explore their relative and absolute energies and aspects of their kinetics and to predict their vibrational spectra to aid possible IR detection. An additional impetus for this work is a recent IR study of matrix-isolated sulfinic acid, ${ }^{3} \mathrm{HSO}_{2} \mathrm{H}$, which provides an opportunity to compare theory with experiment. $\mathrm{H}_{2} \mathrm{SO}_{2}$ has also been proposed as an intermediate in the high-temperature reduction of $\mathrm{SO}_{2}$ by $\mathrm{H}_{2}{ }^{4}$ and some implications for the combustion chemistry of sulfur are considered. One goal of the present work is to add to the relatively small data base of theoretical analyses of sulfur-containing molecules ${ }^{5}$ which are significant in flame and atmospheric chemistry.

\section{Methodology}

Standard ab initio molecular orbital theory, described elsewhere, ${ }^{6}$ was employed in this work. All results were obtained by use of the GAUSSIAN 90 program $^{7}$ on a VAX 6310, a Solbourne 5E/902, and a Cray Y-MP/832 computer. Details of the calculations have been described previously ${ }^{2}$ and are briefly summarized here. A full geometry optimization was carried out for each closed-shell species using the $3-21 G\left(^{*}\right)$ basis set at the spin-restricted HF level. Harmonic vibrational frequencies were calculated for each structure to verify it as a true minimum or a transition state. All frequencies at the HF level were scaled by a standard factor of 0.9 to take account of known inadequacies at this level. Equilibrium geometries were then reoptimized using Møller-Plesset second-order perturbation theory to take electron correlation into account. We denote these results as MP2/3$\left.21 \mathrm{G}^{*}\right)$. Unscaled vibrational frequencies were also calculated at the MP2 level, and the stability of the HF wave function was verified for each structure. Finally, energies at these MP2/3.

(1) Plummer, P. L. M.; Chen, T. S.; Law, K. Y. Atmos. Environ. 1984, 18, 2769 .

(2) Binns, D.; Marshall, P. J. Chem. Phys. 1991, 95, 4940.

(3) Fender, M. A.; Sayed, Y. M.; Prochaska, F. T. J. Phys. Chem. 1991, 95,2811 .

(4) Arutyunov, V. A.; Vedeneev, V. I.; Ushakov, V. A.; Shumova, V. V. Kinet. Catal. 1990, 3I, 6

(5) Basch, H. In The Chemistry of Sulfinic Acids, Esters, and their Derivatives; Wiley: New York, 1990; Chapter 2.

(6) Hehre, W. J.; Radom, L.; Schleyer, P. v. R.; Pople, J. A. Ab Initio Molecular Orbital Theory: Wiley: New York, 1986.

(7) Frisch, M. J.; Head-Gordon, M.; Trucks, G. W.; Foresman, J. B.; Schlegel, H. B.; Raghavachari, K.: Robb, M. A.; Binkley, J. S.; Gonzalez, C.; Defrees, D. J.; Fox, D. J.; Whiteside, R. A.; Seeger, R.; Melius, C. F.; Baker, J.; Martin, R. L.; Kahn, L. R.; Stewart, J. J. P.; Topiol S.; Pople, J. A. Gaussian 90; Gaussian: Pittsburgh, PA, 1990.
21G(*) geometries were calculated using MP4 theory and the larger 6-31G* basis set and are denoted as MP4/6-31G*// MP2/3-21G(*).

\section{Results and Discussion}

The calculated geometries, vibrational frequencies, and energies are listed in Tables I and II. Figure 1 is an illustration of the equilibrium geometries.

Geometries. The HF/3-21G $\left(^{*}\right)$ geometries of sulfone and the A-isomer of sulfinic acid $\left(\mathrm{HSO}_{2} \mathrm{H}\right)$ agree well with the results of Boyd et al. ${ }^{8}$ derived with the smaller STO-3G(*) basis set. Our geometries for $\mathrm{HSOOH}$ and $\mathrm{S}(\mathrm{OH})_{2}$ are similar to those of Plummer et al. ${ }^{1}$ at the STO-3G(*) basis; however, our result for $\mathrm{H}_{2} \mathrm{SOO}$ does not agree. Our S-O bond lengths at both levels of calculation differed markedly from Plummer's result of $2.07 \AA$, while our S-O-O angles contrast Plummer's value of $114.0^{\circ}$ (see Table I). Because our result was derived using a larger basis set, we believe that it is more reliable. Comparison of our geometries for the A-isomer of $\mathrm{HSO}_{2} \mathrm{H}, \mathrm{S}(\mathrm{OH})_{2}$, and sulfone with those calculated by Basch $^{5}$ at the $\mathrm{HF} / 6-31 \mathrm{G}^{*}$ level yield negligible differences; we hence conclude that there is no significant dependence on basis set at the HF level. This is similar to the lack of dependence reported earlier for $\mathrm{HSO}_{2}$ and $\mathrm{HOSO}^{2}$ The similarity of our HF structures to those derived here at the MP2 level further indicates that the inclusion of electron correlation has little effect on geometry for the closed-shell species analyzed here. Sulfinic acid has been observed experimentally by Fender et al. using IR spectroscopy. ${ }^{3}$ For their force-field analysis they assigned standard bond lengths of $1.43(\mathrm{~S}=\mathrm{O}), 1.35(\mathrm{~S}-\mathrm{H})$, and $0.96 \AA$ $(\mathrm{O}-\mathrm{H})$ which lie midway between our calculated HF/3-21G(*) and MP2/3-21G $\left({ }^{*}\right)$ distances, except that our calculations (Table I) suggest the presence of both $S-O$ and $S=O$ bonds.

Vibrational Frequencies. Basch has presented a limited set of harmonic frequencies of stretching modes for the $\mathrm{HSO}_{2} \mathrm{H} \mathrm{A}$ isomer, sulfone, and $\mathrm{S}(\mathrm{OH})_{2}$ at the $\mathrm{HF} / 6-31 \mathrm{G}^{*}$ level. ${ }^{5}$ We find that our frequencies at both the HF and MP2 levels are in close agreement with those reported values. The vibrational spectra of isotopomers of sulfinic acid have been measured by Fender et al. ${ }^{3}$ which provide the only available comparison with theory for the molecules studied here, and the data for $\mathrm{HSO}_{2} \mathrm{H}$ is summarized in Table III. We find that our three highest calculated frequencies (see Table I; the $\mathrm{O}-\mathrm{H}$ stretch at $\nu \approx 3500 \mathrm{~cm}^{-1}$ could not be observed ${ }^{3}$ and is excluded from comparison) are in reasonable agreement with the three largest experimental values of 2591,1209 , and $1093 \mathrm{~cm}^{-1}$ : the mean absolute error for these three frequencies is $7.4 \%$ at the MP2 level and $3.3 \%$ at the HF level. Agreement with the other measured frequencies is much poorer. All calculated $\nu$ are found to be too high. The two lowest calculated frequencies show a mean error of $37 \%$ (MP2) and

(8) Boyd, R. J.; Gupta, A.; Langler, R. F.; Lownie, S. P.; Pincock, J. A. Can. J. Chem. 1980, 58, 331 . 
TABLE I: Ab Initio Geometries and Frequencies

\begin{tabular}{|c|c|c|}
\hline species & calculation & geometry $^{a}$ and frequencies ${ }^{b}$ \\
\hline $\begin{array}{l}\mathrm{HSO}_{2} \mathrm{H} \\
\mathrm{A} \text {-conformer }\end{array}$ & $\mathrm{MP} 2 / 3-21 \mathrm{G}\left({ }^{*}\right)$ & $\begin{array}{l}r\left(\mathrm{SO}_{1}\right)=1.462, r\left(\mathrm{SO}_{2}\right)=1.616, r(\mathrm{OH})=0.938, r(\mathrm{SH})=1.346, \psi=106.3 \\
\quad \vartheta=109.2, \varphi=115.0, \delta\left(\mathrm{H}_{1} \mathrm{O}_{2} \mathrm{SO}_{1}\right)=33.1, \delta\left(\mathrm{H}_{1} \mathrm{SO}_{1} \mathrm{O}_{2}\right)=101.4 \\
\nu=302,458,813,1011,1080,1128,1179,2416,3415 \\
r\left(\mathrm{SO}_{1}\right)=1.487, r\left(\mathrm{SO}_{2}\right)=1.668, r(\mathrm{OH})=1.000, r(\mathrm{SH})=1.380, \psi=107.3, \\
\quad \vartheta=112.0, \varphi=112.3, \delta\left(\mathrm{H}_{1} \mathrm{O}_{2} \mathrm{SO}_{1}\right)=44.4, \delta\left(\mathrm{H}_{1} \mathrm{SO}_{1} \mathrm{O}_{2}\right)=101.2 \\
\nu=311,467,749,1003,1151,1185,1258,2366,3438\end{array}$ \\
\hline $\begin{array}{l}\mathrm{HSO}_{2} \mathrm{H} \\
\text { B-conformer }\end{array}$ & $\mathrm{HF} / 3-21 \mathrm{G}(*)$ & $\begin{array}{l}r\left(\mathrm{SO}_{1}\right)=1.458, r\left(\mathrm{SO}_{2}\right)=1.628, r(\mathrm{OH})=0.970, r(\mathrm{SH})=1.333, \psi=108.6 \\
\vartheta=111.3, \varphi=114.0, \delta\left(\mathrm{H}_{1} \mathrm{O}_{2} \mathrm{SO}_{1}\right)=-79.2, \delta\left(\mathrm{H}_{1} \mathrm{O}_{2} \mathrm{SH}_{2}\right)=171.3 \\
\nu=303,427,807,986,1114,1163,1201,2515,34665\end{array}$ \\
\hline & $\mathrm{MP} 2 / 3-21 \mathrm{G}\left(^{*}\right)$ & $\begin{array}{l}r\left(\mathrm{SO}_{1}\right)=1.488, r\left(\mathrm{SO}_{2}\right)=1.689, r(\mathrm{OH})=0.997, r(\mathrm{SH})=1.363, \psi=109.7 \\
\vartheta=113.2, \varphi=108.7, \delta\left(\mathrm{H}_{1} \mathrm{O}_{2} \mathrm{SO}_{1}\right)=-81.9, \delta\left(\mathrm{H}_{1} \mathrm{O}_{2} \mathrm{SH}_{2}\right)=168.5 \\
\nu=367,470,738,1025,1168,1243,1276,2512,3477\end{array}$ \\
\hline HOSO anion & $\mathrm{HF} / 3-21 \mathrm{G}\left({ }^{*}\right)$ & $\begin{array}{l}r\left(\mathrm{SO}_{1}\right)=1.554, r\left(\mathrm{SO}_{2}\right)=1.732, r(\mathrm{OH})=0.972, \vartheta=108.7, \varphi=108.3, \delta=64.3 \\
\nu=238,408,659,870,1090,3369\end{array}$ \\
\hline & $\mathrm{MP} 2 / 3-21 \mathrm{G}\left(^{*}\right)$ & $\begin{array}{l}r\left(\mathrm{SO}_{1}\right)=1.577, r\left(\mathrm{SO}_{2}\right)=1.826, r(\mathrm{OH})=1.003, \vartheta=112.0, \varphi=103.4, \delta=71.7 \\
\nu=282,413,550,1007,1077,3325\end{array}$ \\
\hline $\mathrm{HSO}_{2}$ anion & $\mathrm{HF} / 3-21 \mathrm{G}\left({ }^{*}\right)$ & $\begin{array}{l}r\left(\mathrm{SO}_{1}\right)=1.493, r\left(\mathrm{SO}_{2}\right)=1.493, r(\mathrm{SH})=1.396, \vartheta=114.8, \psi_{1}=102.0, \psi_{2}=102.0 \\
\nu=468,959,1104,1109,1129,1972\end{array}$ \\
\hline & $\mathrm{MP} 2 / 3-21 \mathrm{G}\left({ }^{*}\right)$ & $\begin{array}{l}r\left(\mathrm{SO}_{1}\right)=1.516, r\left(\mathrm{SO}_{2}\right)=1.516, r(\mathrm{SH})=1.475, \vartheta=115.6, \psi_{1}=102.2, \psi_{2}=102.2 \\
\nu=480,1013,1074,1125,1216,1596\end{array}$ \\
\hline $\mathrm{S}(\mathrm{OH})_{2}$ & $\mathrm{MP} 2 / 3-21 \mathrm{G}(*)$ & $\begin{array}{l}r(\mathrm{SO})=1.634, r(\mathrm{OH})=0.970, \varphi_{1}=113.0, \varphi_{2}=113.0, \vartheta=102.9, \delta\left(\mathrm{H}_{1} \mathrm{O}_{1} \mathrm{SO}_{2}\right)= \\
\quad 80.9, \delta\left(\mathrm{H}_{2} \mathrm{O}_{2} \mathrm{SO}_{1}\right)=80.9 \\
\nu=328,482,516,809,818,1177,1177,3432,3434 \\
r(\mathrm{SO})=1.672, r(\mathrm{OH})=0.998, \varphi_{1}=109.4, \varphi_{2}=109.4, \vartheta=105.2, \delta\left(\mathrm{H}_{1} \mathrm{O}_{1} \mathrm{SO}_{2}\right)= \\
\quad 82.4, \delta\left(\mathrm{H}_{2} \mathrm{O}_{2} \mathrm{SO}_{1}\right)=82.4 \\
\nu=332,566,566,788,801,1254,1259,3440,3441\end{array}$ \\
\hline HSOOH & $\mathrm{HF} / 3-21 \mathrm{G}\left({ }^{*}\right)$ & $\begin{array}{l}r(\mathrm{SH})=1.328, r(\mathrm{SO})=1.654, r(\mathrm{OO})=1.480, r(\mathrm{OH})=0.974, \psi=98.4 \\
\quad \eta=108.6, \gamma=99.0, \delta\left(\mathrm{H}_{2} \mathrm{O}_{2} \mathrm{O}_{1} \mathrm{~S}\right)=165.2, \delta\left(\mathrm{H}_{1} \mathrm{SO}_{1} \mathrm{O}_{2}\right)=82.4 \\
\nu=103,304,370,741,1003,1035,1322,2592,3411\end{array}$ \\
\hline & $\mathrm{MP} 2 / 3-21 \mathrm{G}\left(^{*}\right)$ & $\begin{array}{l}r(\mathrm{SH})=1.344, r(\mathrm{SO})=1.681, r(\mathrm{OO})=1.571, r(\mathrm{OH})=1.005, \psi=99.8 \\
\quad \eta=105.6, \gamma=105.6, \delta\left(\mathrm{H}_{2} \mathrm{O}_{2} \mathrm{O}_{1} \mathrm{~S}\right)=170.9, \delta\left(\mathrm{H}_{1} \mathrm{SO}_{1} \mathrm{O}_{2}\right)=83.8 \\
\nu=95,348,395,718,865,1033,1279,2722,3388\end{array}$ \\
\hline $\mathrm{H}_{2} \mathrm{SOO}$ & $\mathrm{MP} 2 / 3-21 \mathrm{G}\left({ }^{*}\right)$ & $\begin{array}{l}r(\mathrm{OO})=1.618, r(\mathrm{SO})=1.554, r\left(\mathrm{SH}_{1}\right)=1.327, r\left(\mathrm{SH}_{2}\right)=1.338, \eta=89.9 \\
\psi_{1}=105.4, \psi_{2}=103.2, \delta\left(\mathrm{H}_{1} \mathrm{SO}_{2} \mathrm{O}_{1}\right)=68.3, \delta\left(\mathrm{H}_{2} \mathrm{SO}_{2} \mathrm{O}_{1}\right)=-195.1 \\
\nu=244,323,659,828,997,1059,1265,2517,2598 \\
r(\mathrm{OO})=1.641, r(\mathrm{SO})=1.750, r\left(\mathrm{SH}_{1}\right)=1.367, r\left(\mathrm{SH}_{2}\right)=1.367, \eta=62.0 \\
\psi_{1}=132.0, \psi_{2}=91.3, \delta\left(\mathrm{H}_{1} \mathrm{SO}_{2} \mathrm{O}_{1}\right)=55.1, \delta\left(\mathrm{H}_{2} \mathrm{SO}_{2} \mathrm{O}_{1}\right)=-217.6 \\
\nu=273,653,737,1000,1077,1207,1498,2477,2527\end{array}$ \\
\hline sulfone & $\mathrm{HF} / 3-21 \mathrm{G}\left(^{*}\right)$ & $\begin{array}{l}r\left(\mathrm{SO}_{1}\right)=1.429, r\left(\mathrm{SO}_{2}\right)=1.429, r\left(\mathrm{SH}_{1}\right)=1.327, r\left(\mathrm{SH}_{2}\right)=1.327, \psi_{1}=108.4 \\
\psi_{2}=108.4, \vartheta=122.4, \delta\left(\mathrm{H}_{1} \mathrm{SH}_{2} \mathrm{O}_{1}\right)=127.3, \delta\left(\mathrm{H}_{2} \mathrm{SH}_{1} \mathrm{O}_{2}\right)=-127.4 \\
\nu=503,905,1046,1107,1271,1351,1403,2568,2594\end{array}$ \\
\hline & $\mathrm{MP} 2 / 3-21 \mathrm{G}(*)$ & $\begin{array}{l}r\left(\mathrm{SO}_{1}\right)=1.456, r\left(\mathrm{SO}_{2}\right)=1.456, r\left(\mathrm{SH}_{1}\right)=1.357, r\left(\mathrm{SH}_{2}\right)=1.357, \psi_{1}=108.4 \\
\psi_{2}=108.4, \vartheta=123.4, \delta\left(\mathrm{H}_{1} \mathrm{SH}_{2} \mathrm{O}_{1}\right)=128.2, \delta\left(\mathrm{H}_{2} \mathrm{SH}_{1} \mathrm{O}_{2}\right)=-128.2 \\
\nu=511,918,1059,1162,1299,1410,1505,2569,2602\end{array}$ \\
\hline $\begin{array}{l}\mathrm{HSO}_{2} \mathrm{H} \text { to } \\
\quad \mathrm{S}(\mathrm{OH})_{2} \mathrm{TS}\end{array}$ & $\mathrm{MP} 2 / 3-21 G(*)$ & $\begin{array}{l}r\left(\mathrm{SO}_{1}\right)=1.656, r\left(\mathrm{SO}_{2}\right)=1.632, r(\mathrm{OH})=0.971, r(\mathrm{SH})=1.375, \psi=99.9 \\
\quad \vartheta=108.7, \varphi=113.5, \delta\left(\mathrm{H}_{1} \mathrm{O}_{2} \mathrm{SO}_{1}\right)=-79.3, \delta\left(\mathrm{H}_{1} \mathrm{O}_{2} \mathrm{SH}_{2}\right)=-135.3 \\
\nu=2192 i, 265,398,667,833,905,1250,2641,3814 \\
r\left(\mathrm{SO}_{1}\right)=1.633, r\left(\mathrm{SO}_{2}\right)=1.692, r(\mathrm{OH})=0.988, r\left(\mathrm{SH}^{2}\right)=1.373, \psi=96.7 \\
\vartheta=113.9, \varphi=108.8, \delta\left(\mathrm{H}_{1} \mathrm{O}_{2} \mathrm{SO}_{1}\right)=-94.0, \delta\left(\mathrm{H}_{1} \mathrm{O}_{2} \mathrm{SH}_{2}\right)=-152.3 \\
\nu=1896 i, 335,442,712,739,1012,1175,2623,3453\end{array}$ \\
\hline
\end{tabular}

\footnotetext{
${ }^{a}$ Angles are in degrees and distances are in angstroms. See Figure 1 for definitions of coordinates. ${ }^{b}$ Frequencies are given in $\mathrm{cm}^{-1}$. HF results are scaled by a factor of 0.9 , while MP2 results are unscaled.
}

$18.9 \%$ (HF) with respect to the experimental values of 340 and $270 \mathrm{~cm}^{-1}$. The middle-range calculated frequencies corresponding to the experimental values of 762,476 , and $450 \mathrm{~cm}^{-1}$ show even greater deviations from the experimental results. The mean error for these calculations is $77.4 \%(\mathrm{MP} 2)$ and $77.5 \%(\mathrm{HF})$. Presumably these large differences between the observed frequencies and the calculated harmonic frequencies indicate that coupling between normal modes and especially anharmonicity may be important in sulfinic acid. Clearly, the predicted frequencies for the other molecules studied here, other than for the high-frequency stretching modes, must be considered with caution.

Thermochemistry. The order of decreasing stability of the six $\mathrm{H}_{2} \mathrm{SO}_{2}$ isomers with respect to their MP4 energies (including zero-point energies) is $\mathrm{S}(\mathrm{OH})_{2}>\mathrm{HSO}_{2} \mathrm{H}$ B-conformer $\geq \mathrm{HSO}_{2} \mathrm{H}$ A-conformer $>$ sulfone $>\mathrm{HSOOH}>\mathrm{H}_{2} \mathrm{SOO}$. The least stable isomer, $\mathrm{H}_{2} \mathrm{SOO}$, is unlikely to be observed because it is unstable with respect to dissociation to $\mathrm{H}_{2} \mathrm{~S}+\mathrm{O}_{2}$, with an estimated $\Delta H_{0}{ }^{\circ}$ of $-227 \mathrm{~kJ} \mathrm{~mol}^{-1}$. The errors in the calculated frequencies of $\mathrm{HSO}_{2} \mathrm{H}$ lead to an error in the zero-point vibrational energy (ZPE) of about $10 \mathrm{~kJ} \mathrm{~mol}^{-1}$; this must be considered when comparing isomers with energies that lie close together. To test our data, we compared our calculated enthalpies at $0 \mathrm{~K}$ with literature values $^{9}$ for two reactions. The experimental $\Delta H_{0}^{\circ}$ of

$$
\begin{aligned}
& \mathrm{H}_{2} \mathrm{~S}+\mathrm{O}_{2} \rightarrow \mathrm{SO}_{2}+\mathrm{H}_{2} \\
& \mathrm{H}_{2} \mathrm{~S}+\mathrm{O}_{2} \rightarrow \mathrm{H}_{2} \mathrm{O}+\mathrm{SO}
\end{aligned}
$$

are $-277 \pm 1$ and $-216 \pm 2 \mathrm{~kJ} \mathrm{~mol}^{-1}$, respectively. ${ }^{9}$ The calculated values are -274 and $-207 \mathrm{~kJ} \mathrm{~mol}^{-1}$, which correspond to errors of only +3 and $-9 \mathrm{~kJ} \mathrm{~mol}^{-1}$, respectively. On the basis of these differences plus possible errors in ZPE, we suggest error limits of $\pm 20 \mathrm{~kJ} \mathrm{~mol}^{-1}$ for the energies summarized in Table II.

We also considered the reactions

$$
\begin{aligned}
& \mathrm{SO}_{2}+\mathrm{H}_{2} \rightarrow \mathrm{HOS}+\mathrm{OH} \\
& \mathrm{SO}_{2}+\mathrm{H}_{2} \rightarrow \mathrm{HSO}+\mathrm{OH}
\end{aligned}
$$

The heats of formation of HSO and HOS have not been measured, but Benson ${ }^{10}$ has estimated $\Delta H_{\mathrm{f}}^{\circ}{ }_{298}(\mathrm{HSO})=-21 \pm 17 \mathrm{~kJ} \mathrm{~mol}^{-1}$,

(9) Chase, M. W., Jr.; Davies, C. A.; Downey, J. R.; Frurip, D. J.; McDonald, R. A.; Syverud, A. N. JANAF Thermochemical Tables, 3rd ed.; J. Phys. Chem. Ref. Data 1985, 14 (Suppl. No. 1). 
TABLE II: Ab Initio Energies

\begin{tabular}{|c|c|c|c|}
\hline species & calculation & $\begin{array}{c}\text { energy, } \\
\text { au }\end{array}$ & $\begin{array}{c}\Delta H_{0} \\
\mathrm{~kJ} \\
\mathrm{~mol}^{-1}\end{array}$ \\
\hline $\begin{array}{l}\mathrm{HSO}_{2} \mathrm{H} \\
\mathrm{A} \text {-conformer }\end{array}$ & $\begin{array}{l}\mathrm{HF} / 3-21 \mathrm{G}\left(^{*}\right) \\
\text { MP2/3-21G(*) } \\
\text { MP4/6-31G*//MP2/3-21G(*) }\end{array}$ & $\begin{array}{l}-545.6396 \\
-546.0230 \\
-548.8065\end{array}$ & \\
\hline $\begin{array}{l}\mathrm{HSO}_{2} \mathrm{H} \\
\text { B-conformer }\end{array}$ & $\begin{array}{l}\text { HF/3-21G(*) } \\
\text { MP2/3-21G(*) } \\
\text { MP4/6-31G*//MP2/3-21G(*) }\end{array}$ & $\begin{array}{l}-545.6375 \\
-546.0244 \\
-548.8070\end{array}$ & \\
\hline HOSO anion & $\begin{array}{l}\mathrm{HF} / 3-21 \mathrm{G}\left({ }^{*}\right) \\
\text { MP2 } 2 / 3-21 \mathrm{G}\left({ }^{*}\right) \\
\text { MP4/6-31G*//MP2/3-21G(*) }\end{array}$ & $\begin{array}{l}-545.0566 \\
-545.4329 \\
-548.2483\end{array}$ & 1467 \\
\hline $\mathrm{HSO}_{2}$ anion & $\begin{array}{l}\mathrm{HF} / 3-21 \mathrm{G}\left({ }^{*}\right) \\
\left.\text { MP2 } 2 / 3-21{ }^{*}\right) \\
\text { MP4/6-31G*//MP2/3-21G(*) }\end{array}$ & $\begin{array}{l}-545.0588 \\
-545.4397 \\
-548.2568\end{array}$ & 1440 \\
\hline $\mathrm{S}(\mathrm{OH})_{2}$ & $\begin{array}{l}\mathrm{HF} / 3-21 \mathrm{G}\left({ }^{*}\right) \\
\mathrm{MP} 2 / 3-21 \mathrm{G}\left({ }^{*}\right) \\
\text { MP4/6-31G* }\end{array}$ & $\begin{array}{l}-545.6757 \\
-546.0484 \\
-548.8238\end{array}$ & -56 \\
\hline HSOOH & $\begin{array}{l}\text { HF/3-21G(*) } \\
\text { MP2/3-21G(*) } \\
\text { MP4/6-31G*//MP2/3-21G(*) }\end{array}$ & $\begin{array}{l}-545.5944 \\
-545.9784 \\
-548.7456\end{array}$ & 161 \\
\hline $\mathrm{H}_{2} \mathrm{SOO}$ & $\begin{array}{l}\mathrm{HF} / 3-21 \mathrm{G}\left(^{*}\right) \\
\text { MP2/3-21G(*) } \\
\text { MP4/6-31G } / / \text { MP2/3-21G(*) }\end{array}$ & $\begin{array}{l}-545.4951 \\
-545.8889 \\
-548.6546\end{array}$ & 400 \\
\hline sulfone & $\begin{array}{l}\mathrm{HF} / 3-21 \mathrm{G}\left({ }^{*}\right) \\
\mathrm{MP} 2 / 3-21 \mathrm{G}\left({ }^{*}\right) \\
\text { MP4/6-31G*//MP2/3-21G(*) }\end{array}$ & $\begin{array}{l}-545.6107 \\
-546.0052 \\
-548.7771\end{array}$ & 78 \\
\hline $\mathrm{H}_{2}$ & $\begin{array}{l}\mathrm{HF} / 3-21 \mathrm{G}\left(^{*}\right) \\
\text { MP2/3-21G(*) } \\
\text { MP4/6-31G*//MP2/3-21G(*) }\end{array}$ & $\begin{array}{l}-1.1230 \\
-1.1403 \\
-1.1406\end{array}$ & \\
\hline $\mathrm{O}_{2}$ & $\begin{array}{l}\mathrm{HF} / 3-21 \mathrm{G}\left(^{*}\right) \\
\left.\mathrm{MP} 2 / 3-21 \mathrm{G}^{*}\right) \\
\mathrm{MP} 4 / 6-31 \mathrm{G}^{*} / / \mathrm{MP} 2 / 3-21 \mathrm{G}\left({ }^{*}\right)\end{array}$ & $\begin{array}{l}-148.7691 \\
-149.0344 \\
-149.9449\end{array}$ & \\
\hline $\mathrm{H}_{2} \mathrm{O}$ & $\begin{array}{l}\mathrm{HF} / 3-21 \mathrm{G}\left({ }^{*}\right) \\
\mathrm{MP} 2 / 3-21 \mathrm{G}\left({ }^{*}\right) \\
\mathrm{MP} 4 / 6-31 \mathrm{G}^{*} / / \mathrm{MP} 2 / 3-21 \mathrm{G}\left(^{*}\right)\end{array}$ & $\begin{array}{l}-75.5850 \\
-75.7096 \\
-76.1863\end{array}$ & \\
\hline $\mathrm{H}_{2} \mathrm{~S}$ & $\begin{array}{l}\mathrm{HF} / 3-21 \mathrm{G}\left({ }^{*}\right) \\
\text { MP2/3-21G(*) } \\
\text { MP4/6-31G*//MP2/3-21G(*) }\end{array}$ & $\begin{array}{l}-396.8196 \\
-396.9563 \\
-398.7962\end{array}$ & \\
\hline HOS & $\begin{array}{l}\mathrm{HF} / 3-21 \mathrm{G}\left(^{*}\right) \\
\text { MP2/3-21G(*) } \\
\text { MP4/6-31G*//MP2/3-21G(*) }\end{array}$ & $\begin{array}{l}-470.6473 \\
-470.8838 \\
-473.1991\end{array}$ & \\
\hline HSO & $\begin{array}{l}\mathrm{HF} / 3-21 \mathrm{G}\left(^{*}\right) \\
\text { MP2/3-21G(*) } \\
\text { MP4/6-31G } / / \text { MP2/3-21G(*) }\end{array}$ & $\begin{array}{l}-470.6430 \\
-470.8725 \\
-473.2015\end{array}$ & \\
\hline $\begin{array}{l}\mathrm{HSO}_{2} \mathrm{H} \text { to } \\
\quad \mathrm{S}(\mathrm{OH})_{2} \mathrm{TS}\end{array}$ & $\begin{array}{l}\mathrm{HF} / 3-21 \mathrm{G}\left(^{*}\right) \\
\text { MP2/3-21G(*) } \\
\text { MP4/6-31G*//MP2/3-21G(*) }\end{array}$ & $\begin{array}{l}-545.5356 \\
-545.9396 \\
-548.7587\end{array}$ & 190 \\
\hline $\begin{array}{l}\mathrm{HSO}_{2} \mathrm{H} \\
\text { conformer-A } \\
\text { to -B TS }\end{array}$ & $\begin{array}{l}\mathrm{HF} / 3-21 \mathrm{G}\left({ }^{*}\right) \\
\text { MP2/3-21G(*) } \\
\text { MP4/6-31G*//MP2/3-21G(*) }\end{array}$ & $\begin{array}{l}-545.6253 \\
-546.0094 \\
-548.7968\end{array}$ & 27 \\
\hline \multicolumn{4}{|c|}{$\begin{array}{l}\text { a Enthalpy at } 0 \mathrm{~K} \text { relative to } \mathrm{HSO}_{2} \mathrm{H} \text {, B-conformer using } \mathrm{MP} 4 / 6 \text { - } \\
31 \mathrm{G}^{*} \text { energies combined with zero-point energies calculated at the } \\
\mathrm{MP} 2 / 3-21 \mathrm{G}\left({ }^{*}\right) \text { level. }\end{array}$} \\
\hline freq, $\mathrm{cm}^{-1}$ & freq, $\mathrm{cm}^{-1}$ & assig & \\
\hline $\begin{array}{r}2591 \\
1209 \\
1093 \\
762\end{array}$ & $\begin{array}{l}\text { H-S stretch } \\
\text { S-O stretch } \\
\text { S-O-H bend } \\
\text { S-OH stretch }\end{array}$ & $\begin{array}{l}\mathrm{H}-\mathrm{S}-\mathrm{OH} \\
\mathrm{O}-\mathrm{S}-\mathrm{OH} \\
\mathrm{S}-\mathrm{O} \text { wag } \\
\text { torsion }\end{array}$ & \\
\hline
\end{tabular}

and $\Delta H_{\mathrm{f}}{ }^{\circ} 298(\mathrm{HOS})=21 \pm 17 \mathrm{~kJ} \mathrm{~mol}^{-1}$. We have estimated $\Delta H_{\mathrm{f}}^{\circ}{ }_{0}$ for HSO and HOS from $\Delta H_{0}{ }^{\circ}$ of reactions 3 and 4 combined with the experimental $\Delta H_{\mathrm{f}}^{\circ}{ }_{0}$ of $\mathrm{SO}_{2}$ and $\mathrm{OH}$ : our results are $\Delta H_{\mathrm{f}}{ }^{\circ}{ }_{0}(\mathrm{HSO})=-21 \mathrm{~kJ} \mathrm{~mol}^{-1}$ and $\Delta H_{\mathrm{f}}{ }^{\circ}{ }_{0}(\mathrm{HOS})=-1 \mathrm{~kJ} \mathrm{~mol}^{-1}$.

We have investigated the gas-phase acidity of sulfinic acid and our results are in qualitative accord with an earlier study by Boyd et al. ${ }^{8}$ Our estimated enthalpies at the MP4 level, including ZPE, for the reactions

$$
\begin{gathered}
\mathrm{HSO}_{2} \mathrm{H} \rightarrow \mathrm{HSO}_{2}^{-}+\mathrm{H}^{+} \\
\mathrm{HSO}_{2} \mathrm{H} \rightarrow \mathrm{HOSO}^{-}+\mathrm{H}^{+}
\end{gathered}
$$

(10) Benson, S. W. Chem. Rev. 1978, 78, 23.

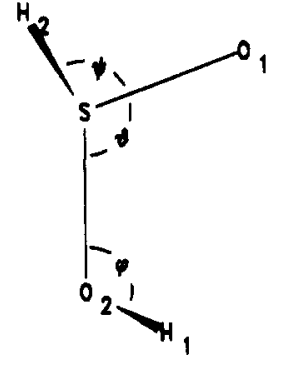

A

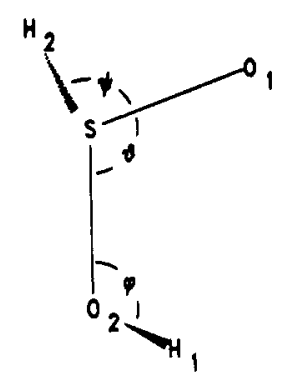

B

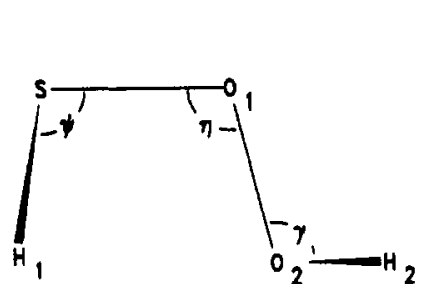

C

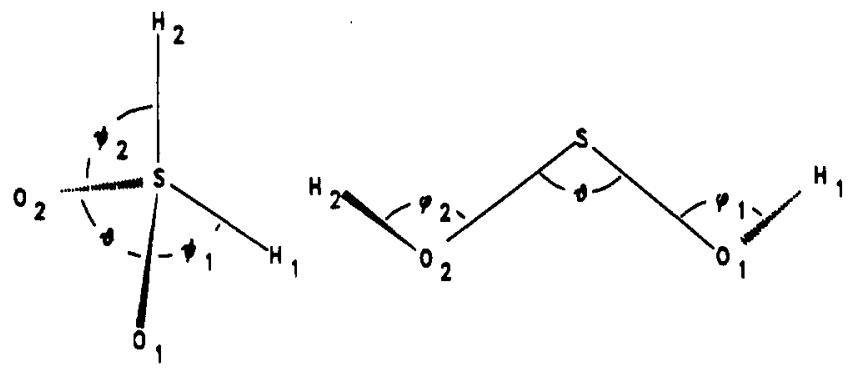

D

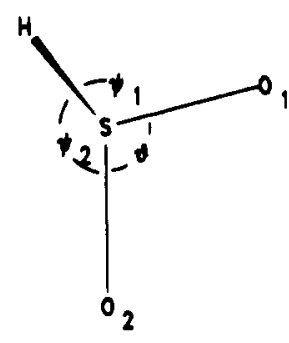

E

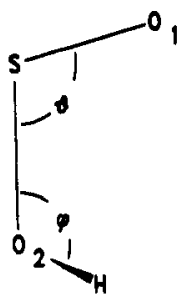

$\mathrm{F}$

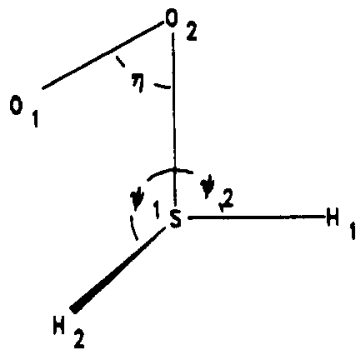

G
Figure 1. Sketch of molecular structures showing definitions of bond angles: (A, B) two conformers of sulfinic acid, $\mathrm{HSO}_{2} \mathrm{H}$; (C) $\mathrm{HSOOH}$; (D) sulfone; (E) $\mathrm{HSO}_{2}^{-}$anion; (F) $\mathrm{HOSO}^{-}$anion; (G) $\mathrm{H}_{2} \mathrm{SOO}$; (H) $\mathrm{S}(\mathrm{OH})_{2}$.

are 1444 and $1467 \mathrm{~kJ} \mathrm{~mol}^{-1}$, respectively. The slightly greater stability of the dissociation product in (5) is consistent with the charge distribution of $\mathrm{HSO}_{2} \mathrm{H}$ calculated at the $\mathrm{HF}$ level: $\mathrm{H}_{1}$ (see Figure 1) has a net atomic charge of +0.472 , while $\mathrm{H}_{2}$ has a much smaller positive charge of +0.047 .

A transition state for internal rotation of the A-conformer around the $\mathrm{S}-\mathrm{O}_{2}$ bond to form the B-conformer has been characterized: there is a rotational barrier of $27 \mathrm{~kJ} \mathrm{~mol}^{-1}$. At high temperatures, the small height of this barrier should allow the molecule to exist as a free rotor.

Chemistry in Solid Ar Matrices. It is interesting to note that although $\mathrm{S}(\mathrm{OH})_{2}$ is $56 \mathrm{~kJ} \mathrm{~mol}^{-1}$ more stable than $\mathrm{HSO}_{2} \mathrm{H}$, only $\mathrm{HSO}_{2} \mathrm{H}$ has been observed experimentally. ${ }^{3}$ To explain this, the thermodynamics of successive addition of $\mathrm{H}$ atoms to $\mathrm{SO}_{2}$ was investigated (see Figure 2). The initial $\mathrm{H}$ atom addition may follow path A or B to form one of the products $\mathrm{HOSO}$ or $\mathrm{HSO}_{2}$. 


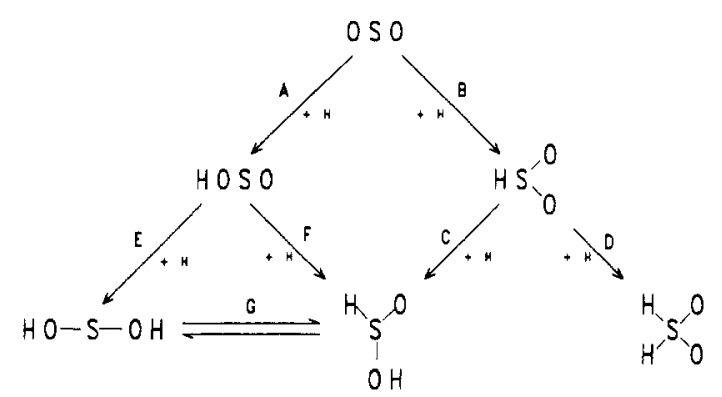

Figure 2. Possible products arising from the sequential addition of $\mathrm{H}$ atoms to $\mathrm{SO}_{2}$

TABLE IV: Possible Dissociation Products of $\mathrm{HSO}_{2} \mathrm{H}$

\begin{tabular}{lccc}
\hline \multicolumn{1}{c}{ products } & $\Delta H^{\circ}{ }_{0}{ }^{a} \mathrm{~kJ} \mathrm{~mol}^{-1}$ & products & $\Delta H^{\circ}{ }_{0},{ }^{a} \mathrm{~kJ} \mathrm{~mol}^{-1}$ \\
\hline $\mathrm{SO}_{2}+\mathrm{H}_{2}$ & -101 & $\mathrm{H}_{2} \mathrm{~S}+\mathrm{O}_{2}$ & 173 \\
$\mathrm{H}_{2} \mathrm{O}+\mathrm{SO}$ & -34 & $\mathrm{HSO}_{2}+\mathrm{H}$ & 247 \\
$\mathrm{HOSO}+\mathrm{H}$ & 125 & &
\end{tabular}

${ }^{a}$ Enthalpies at $0 \mathrm{~K}$, relative to $\mathrm{HSO}_{2} \mathrm{H}$, using MP4/6-31G* energies combined with zero-point energies calculated at the MP2/3-21G(*) level.

These reactions were investigated in an earlier study, ${ }^{2}$ and the formation of the HOSO radical (path A) was found to be more exothermic than addition to form $\mathrm{HSO}_{2}$ (path B). However, it was also indicated in that work that although HOSO is more stable, there is a lower activation barrier to formation of $\mathrm{HSO}_{2}$, so that path $\mathrm{B}$ is favored kinetically. Addition of a second $\mathrm{H}$ atom may follow paths $\mathrm{C}$ or D to $\mathrm{HSO}_{2} \mathrm{H}$. Path $\mathrm{C}$ is the thermodynamically controlled route, because $\mathrm{HSO}_{2} \mathrm{H}$ is $78 \mathrm{~kJ} \mathrm{~mol}^{-1}$ more stable than sulfone. Thus, we suggest that $\mathrm{HSO}_{2} \mathrm{H}$ is formed via steps $B$ and $C$. We verified the absence of any significant barrier to step $C$ at the HF/3-21G(*) and MP2/3-21G(*) levels of calculation. Similarly, steps $\mathrm{E}$ and $\mathrm{F}$ also have no significant kinetic barriers, but the initial step A to formation of HOSO is kinetically unfavorable as noted above.

We also considered formation of $\mathrm{S}(\mathrm{OH})_{2}$ by isomerization of the less stable $\mathrm{HSO}_{2} \mathrm{H}$ (path $\mathrm{G}$ ). We have characterized a transition state for path $\mathrm{G}$, with a barrier of approximately 190 $\mathrm{kJ} \mathrm{mol}^{-1}$ (see Tables I and II). Transition-state theory was applied to investigate the kinetics of the isomerization of sulfinic acid. First-order rate constants $k$ for the high-pressure limit were calculated for the temperature range $298-2000 \mathrm{~K}$, and the fit to the Arrhenius plot yields $k \approx 3.0 \times 10^{13} \exp \left(-2.3 \times 10^{4} \mathrm{~K} / T\right)$ $\mathrm{s}^{-1}$. Thus, equilibration of $\mathrm{HSO}_{2} \mathrm{H}$ and $\mathrm{S}(\mathrm{OH})_{2}$ at low temperatures is negligible.

High-Temperature Chemistry. Possible decomposition products of sulfinic acid, together with the predicted $\Delta H_{0}{ }^{\circ}$ for each channel, are summarized in Table IV. $\mathrm{HSO}_{2} \mathrm{H}$ is seen to be thermodynamically unstable with respect to both $\mathrm{SO}_{2}+\mathrm{H}_{2}$ and $\mathrm{H}_{2} \mathrm{O}+$ $\mathrm{SO}$, although there may be kinetic barriers to these decompositions. Arutyunov et al. ${ }^{4}$ suggested a species of stoichiometry $\mathrm{H}_{2} \mathrm{SO}_{2}$ might be formed by the process

$$
\mathrm{H}_{2}+\mathrm{HSO}_{2} \rightarrow \mathrm{H}_{2} \mathrm{SO}_{2}+\mathrm{H}
$$

at $1000-1200 \mathrm{~K}$, but combination of the $\mathrm{H}_{2}$ bond energy ${ }^{9}$ of 432 $\mathrm{kJ} \mathrm{mol}^{-1}$ with the data of Table IV shows $\Delta H_{0}{ }^{\circ}$ to be about +185 $\mathrm{kJ} \mathrm{mol}^{-1}$. The analogous reaction of the more stable HOSO has $\Delta H_{0}{ }^{\circ} \approx+307 \mathrm{~kJ} \mathrm{~mol}^{-1}$, so reaction 7 is probably insignificant. An alternative formation pathway for $\mathrm{HSO}_{2} \mathrm{H}$ in flames is by $\mathrm{H}$ atom addition to HOSO, as analyzed in the previous section, which is exothermic (Table IV) and appears to have no energy barrier. This would provide a reaction that competes with the second step of the cycle

$$
\begin{aligned}
\mathrm{H}+\mathrm{SO}_{2}(+\mathrm{M}) & \rightarrow \mathrm{HOSO}(+\mathrm{M}) \\
\mathrm{H}+\mathrm{HOSO} & \rightarrow \mathrm{H}_{2}+\mathrm{SO}_{2}
\end{aligned}
$$

which has been invoked to explain the $\mathrm{SO}_{2}$-catalyzed recombination of $\mathrm{H}$ atoms in flames. ${ }^{11}$ The subsequent high-temperature chemistry of sulfinic acid, whether through reactions with other flame species or dissociation e.g. to $\mathrm{H}_{2}+\mathrm{SO}_{2}$, remains to be investigated.

\section{Conclusions}

On the basis of $a b$ initio calculations, we have compared the stability of sulfinic acid with several of its isomers. The calculated vibrational frequencies may aid in the identification of these species by IR spectroscopy. The $\mathrm{S}(\mathrm{OH})_{2}$ molecule is the most stable isomer, followed by sulfinic acid, which may exist in two possible conformations that differ by only $1 \mathrm{~kJ} \mathrm{~mol}^{-1}$. We have located a small barrier to internal rotation of $27 \mathrm{~kJ} \mathrm{~mol}^{-1}$, and we have also characterized a transition state from sulfinic acid, which has been observed, to the more stable $\mathrm{S}(\mathrm{OH})_{2}$, which has not been experimentally detected. The barrier to isomerization of approximately $190 \mathrm{~kJ} \mathrm{~mol}^{-1}$ prevents formation of the thermodynamic product at low temperatures.

Acknowledgment. D.L. thanks the Department of Chemistry and the Texas Academy of Mathematics and Science for a Minority Student Research Fellowship. This work was supported by the Robert A. Welch Foundation (Grant B-1174), the UNT Organized Research Fund, and the NSF Pittsburgh Supercomputing Center (Grant CHE900059P).

(11) Kallend, A. S. Combust. Flame 1972, 19, 227. 